\section{Isolated hemolytic anemia: an unusual manifestation of occult malignancy}

\author{
Matthew J. Butler, ${ }^{1}$ Ming Yin, ${ }^{1}$ \\ Fahd Quddus² \\ 1'Department of Internal Medicine, \\ 2Department of Hematology and \\ Oncology, Geisinger Medical Center, \\ Danville, PA, USA
}

\begin{abstract}
Hemolysis is an uncommon and usually late complication of malignancy, and very rarely the presenting feature. Cancer-associated hemolysis may be immune-mediated, or may result from thrombotic microangiopathy accompanied by thrombocytopenia. We describe an unusual case of isolated hemolysis in the setting of occult metastatic breast cancer. The patient initially presented with symptomatic anemia, with evidence of hemolysis but with negative direct antiglobulin testing and a normal platelet count. Subsequent investigation discovered metastatic adenocarcinoma of the breast involving bone marrow. Hemolysis worsened despite initial treatment with cytotoxic chemotherapy and a trial of corticosteroids, but later resolved with aromatase inhibitor therapy.
\end{abstract}

\section{Introduction}

Thrombotic microangiopathy is an uncommon but well-described complication of solid malignancies, ${ }^{1}$ and has been reported in association with gastric, ${ }^{2-4}$ breast, ${ }^{5,6}$ prostate, ${ }^{7}$ and other cancers. ${ }^{8}$ It typically occurs late in the course of disease, in the setting of malignant infiltration of bone marrow, ${ }^{9}$ or as a side-effect of chemotherapeutic agents. The resulting microangiopathic hemolytic anemia (MAHA) normally presents with marked thrombocytopenia, and can be clinically indistinguishable from thrombotic thrombocytopenic purpura (TTP) or from the hemolytic uremic syndrome (HUS). Unlike classic TTP, cancer-associated MAHA may have normal or variable levels of ADAMTS13 activity, ${ }^{5,10}$ and does not respond to therapeutic plasma exchange. ${ }^{11}$ Like TTP-HUS, cancerassociated MAHA involves platelet aggregation and thrombus formation, resulting in platelet consumption, impaired microvascular blood flow, and mechanical destruction of erythrocytes. Since this process is associated with metastatic involvement of bone marrow, the culprit disease is typically advanced and readily apparent, but rarely MAHA is the presenting feature of an undiagnosed malignancy. ${ }^{12,13}$ In some instances a patient will be misdiagnosed with primary TTP, and subsequently found to harbor an underlying cancer. ${ }^{14}$ In $75 \%$ of cases, cancer-associated MAHA will respond to medical therapy directed at the underlying tumor. ${ }^{8}$

The other described form of malignancyassociated hemolysis is a paraneoplastic form of autoimmune hemolytic anemia (AIHA), reported commonly in the setting of chronic lymphocytic leukemia, ${ }^{15,16}$ and rarely in association with solid tumors. ${ }^{17,18}$ The direct Coombs or direct antiglobulin test (DAT) is considered sensitive for AIHA, though DAT can be negative in patients with low levels of the offending autoantibody. ${ }^{19}$ The hemolytic process may or may not respond to corticosteroid therapy, but cancer-associated AIHA that is refractory to steroids frequently resolves with resection of the primary tumor. $^{18}$

\section{Case Report}

A 75-year-old female presented complaining of progressive fatigue and dyspnea over several months. Physical examination was significant only for pallor. Palpable splenomegaly was absent. Laboratory studies showed macrocytic anemia with a hemoglobin of $8.2 \mathrm{~g} / \mathrm{dL}$, hematocrit of $25.3 \%$, and mean corpuscular volume of $103.7 \mathrm{fL}$; these values had been within normal limits one year prior. Her platelet count was $188,000 / \mathrm{mL}$ and white blood cell count was $10,000 / \mathrm{mL}$, both normal, as were tests of renal and hepatic function, coagulation, and micronutrient levels including iron, folate, and vitamin B-12. The reticulocyte index was 2.4 , but only 1.6 after correction for maturation factor, showing a suboptimal response to anemia. Erythrocyte sedimentation rate was elevated at $94 \mathrm{~mm} / \mathrm{h}$. Lactate dehydrogenase was elevated at 549 IU/L (normal range 90-250) and haptoglobin was undetectable, consistent with a hemolytic process. D-dimer was mildly elevated at $1.04 \mathrm{\mu g} / \mathrm{mL}$, and fibrinogen was within normal limits at $363 \mathrm{mg} / \mathrm{dL}$. A peripheral blood smear contained schistocytes and spherocytes, suggesting hemolysis, and also showed leukoerythroblastosis, with polychromasia, nucleated red blood cells, and immature granulocytes (Figure 1). DAT was negative on two separate occasions, a cold agglutinin assay was negative, and hemoglobin electrophoresis and serum protein electrophoresis were normal. Flow cytometry found no evidence of paroxysmal nocturnal hemoglobinuria. Abdominal
Correspondence: Matthew James Butler, Internal Medicine Residency Program, Geisinger Medical Center, 100 North Academy Avenue, Danville, PA, 17822, USA.

Tel. +1.812.774.7287 - Fax: +1.570.271.6141

E-mail: mjbutler1@geisinger.edu

Key words: hemolysis, hemolytic anemia, malignancy, cancer.

Contributions: the authors contributed equally.

Conflict of interests: the authors declare no potential conflict of interests.

Received for publication: 6 November 2013. Revision received: 24 December 2013.

Accepted for publication: 12 January 2014.

This work is licensed under a Creative Commons Attribution NonCommercial 3.0 License (CC BYNC 3.0).

(C) Copyright M. J. Butler et al., 2014

Licensee PAGEPress, Italy

Hematology Reports 2014; 6:5159

doi:10.4081/hr.2014.5159

ultrasound showed a sonographically normal spleen. As part of the patient's workup for anemia, upper endoscopy was performed and found no source of bleeding, but did recover a sessile $10 \mathrm{~mm}$ polyp from the gastric fundus, which was found to contain a microscopic focus of adenocarcinoma. A bone marrow biopsy revealed poorly differentiated signet ring adenocarcinoma, raising concern for a metastatic gastric cancer (Figure 2). At this point cytotoxic therapy with capecitabine was initiated. The patient's anemia continued to worsen, requiring several red blood cell transfusions, and she was given darbepoetin alfa to promote erythropoiesis. She developed an indirect hyperbilirubinemia of $2.7 \mathrm{mg} / \mathrm{dL}$, while maintaining normal platelet counts and appropriate reticulocytosis, with a corrected reticulocyte index as high as 8.4. However, her anemia did not improve. A six-week trial of corticosteroids, given as empiric treatment for possible DAT-negative AIHA, similarly produced no improvement in anemia or in laboratory markers of hemolysis.

After starting treatment for presumed gastric cancer, a palpable breast mass was discovered. Biopsy revealed signet ring adenocarcinoma that was histologically similar to both bone marrow and gastric polyp findings, with strong estrogen receptor positivity (Figure 3). Additional immunohistochemical staining of the initial bone marrow sample was performed at this time, and was positive for estrogen receptor, E-cadherin, and GATA3 , consistent with primary breast cancer. PETCT imaging showed diffusely mottled osseous structures consistent with an infiltrative 
process, but no other metastatic foci. In light of these findings, corticosteroids and chemotherapy were stopped and aromatase inhibition with anastrozole was initiated. This was followed by a rapid improvement in anemia, allowing blood transfusions and darbepoetin alfa treatments to be stopped. The patient survived 28 months from initial presentation with no evidence of recurrent hemolysis, before succumbing to progression of breast cancer.

\section{Discussion}

This is an unusual case of isolated intravascular hemolysis causing transfusion-dependent symptomatic anemia, in the setting of estrogen receptor positive breast adenocarcinoma metastatic to bone marrow. A causal link between the hemolytic process and the underlying malignancy is supported by the dramatic resolution of hemolysis after the initiation of cancer-directed hormone therapy. Although reticulocytosis at presentation was muted, it responded appropriately to an erythropoiesis-stimulating agent, suggesting that the anemia was primarily attributable to erythrocyte destruction rather than impaired production. Key features of thrombotic microangiopathy were absent: the patient's platelet count remained within the normal range, and she did not develop renal dysfunction or neurologic symptoms. However, the presence of numerous schistocytes in peripheral blood is highly suggestive of mechanical hemolysis, especially in the setting of malignant infiltration of bone marrow. Negative DATs and nonresponse to corticosteroids argue against paraneoplastic AIHA. The hemolytic process observed in this case therefore appears to represent an atypical form of MAHA without associated thrombocytopenia. The reason for the preserved platelet count is unclear.

Despite the advanced stage of the patient's breast cancer, anemia was the only symptom at the time of presentation. The malignant signet ring cells found in bone marrow were wrongly deemed to represent primary gastric cancer; staining for hormone receptors could have provided important diagnostic information, and should have been performed at the time of the first biopsy. The initial misdiagnosis caused a delay in the initiation of estrogen suppression, which allowed observation of ongoing hemolysis as a manifestation of the uncontrolled cancer. Subsequently, there was marked improvement in response to aromatase inhibitor therapy, demonstrating that hemolysis in malignancy may be reversible with effective treatment of the underlying disease.

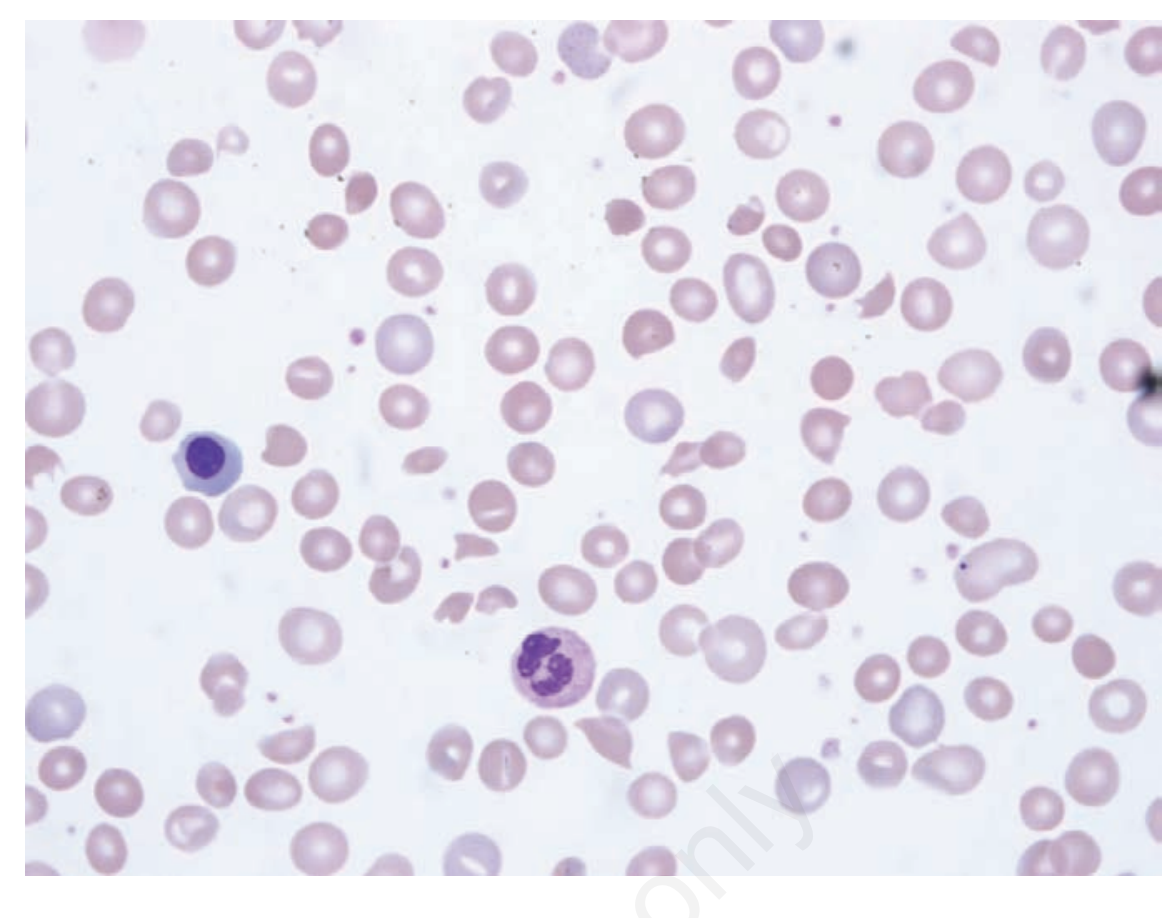

Figure 1. Peripheral blood smear with evidence of intravascular hemolysis.

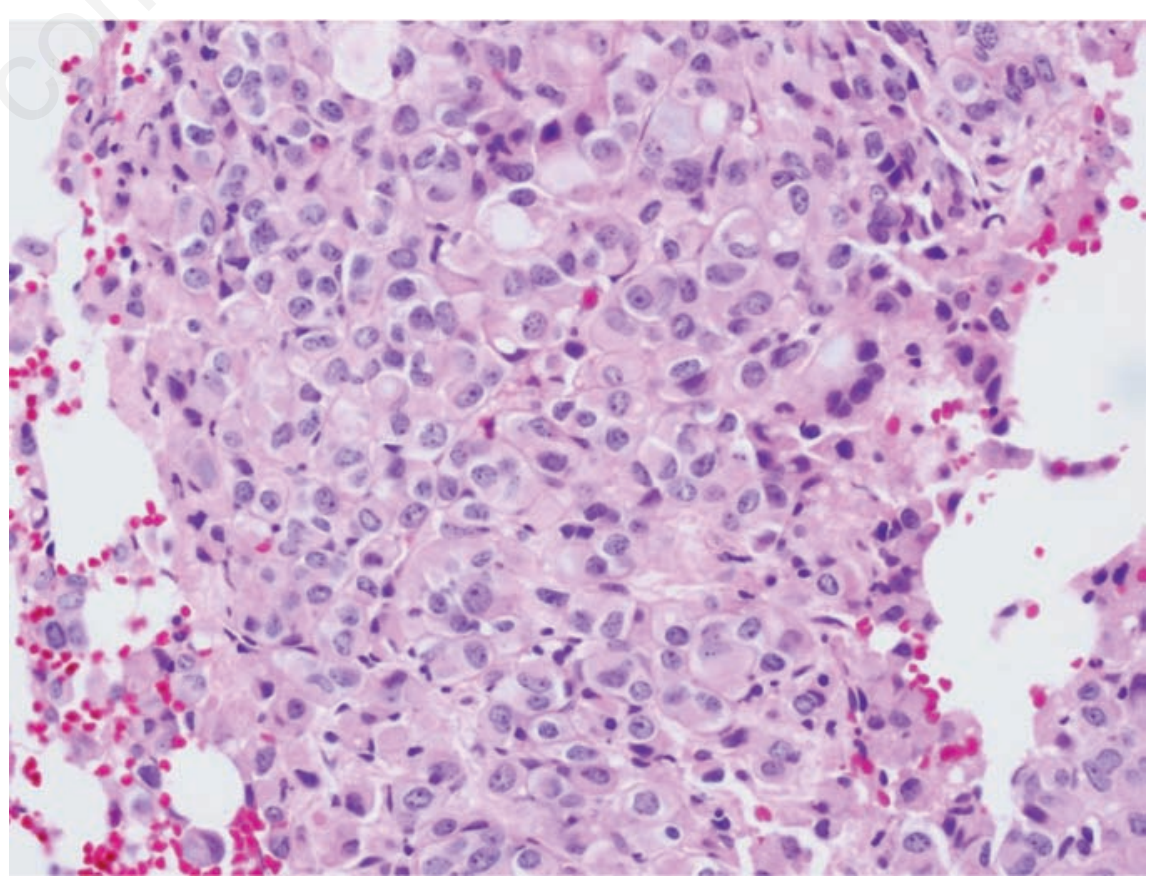

Figure 2. Bone marrow biopsy with focal infiltration of adenocarcinoma. 


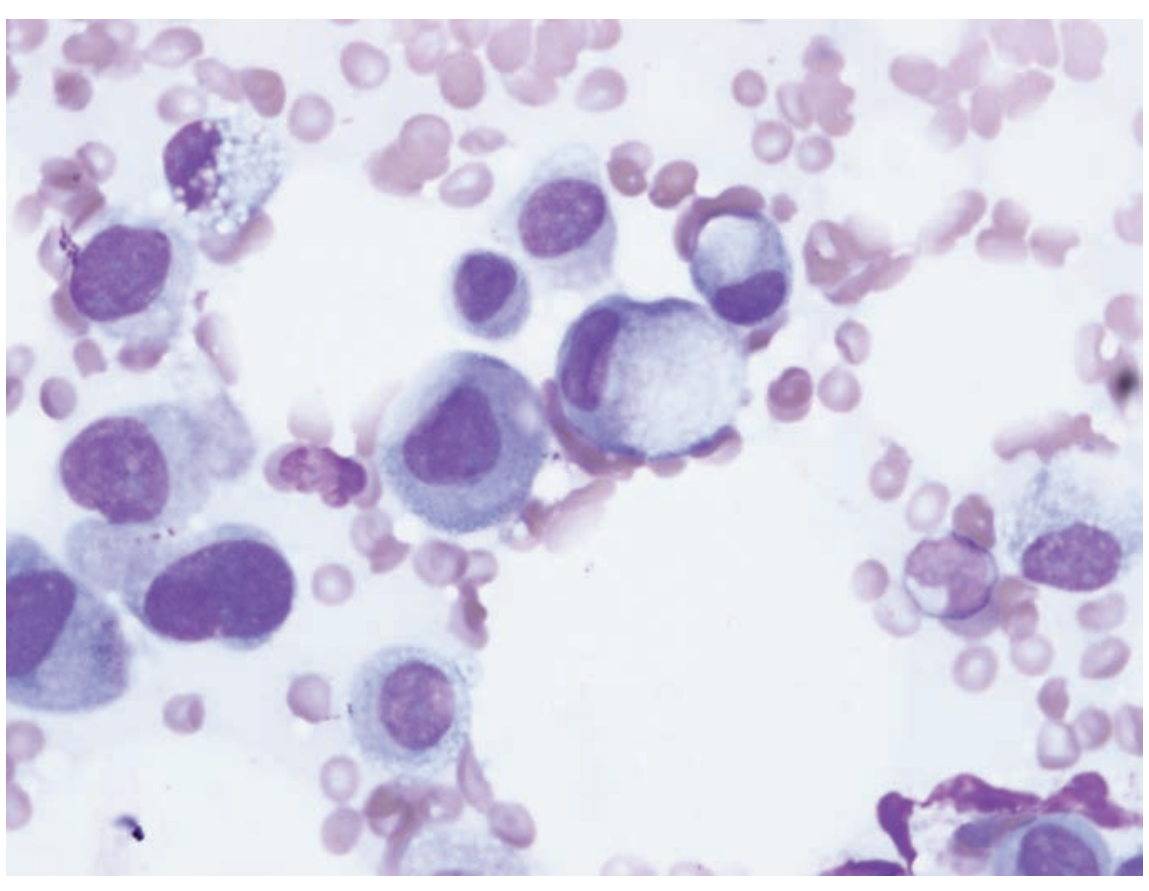

Figure 3. Wright's stain of bone marrow biopsy demonstrating signet ring cells.

\section{Conclusions}

Hemolytic anemia of unclear etiology, with or without thrombocytopenia, should trigger clinical suspicion for occult malignancy and consideration for bone marrow biopsy. In a patient with known cancer, hemolysis should raise concern for disseminated disease with bone marrow involvement, and is a poor prognostic sign. However, such hemolysis may resolve in response to cancer-directed therapy.

\section{References}

1. Kwaan HC, Gordon LI. Thrombotic microangiopathy in the cancer patient. Acta Haematologica 2001;106:52-6.

2. Alexopoulou A, Dourakis SP, Nomikou E. Case of thrombotic thrombocytopenic purpura associated with disseminated gastric cancer. Am J Clin Oncol 2002;25:632.

3. Ali N, Kamran N, Adil S, Pervez S. Metastatic signet ring gastric adenocarcinoma presenting with microangiopathic hemolytic anemia. Indian J Gastroenterol 2007;26:185-6.

4. Kaidar-Person 0, Nasrallah H, Haim N, et al. Disseminated carcinoma diagnosed by bone marrow biopsy in patients with microangiopathic hemolytic anemia and thrombocytopenia: a report of two cases with gastric cancer and a review of the literature. J Gastrointest Cancer 2011;42: 123-6.

5. Regierer AC, Kuehnhardt D, Schulz CO, et al. Breast cancer-associated thrombotic microangiopathy. Breast Care (Basel) 2011;6:441-5.

6. Himmelmann A, Schefer $H$. Microangiopathic haemolytic anaemia in a patient with metastatic breast cancer. $\mathrm{Br}$ J Haematol 2009;146:231.

7. Ramos R, Lopes F, Rodrigues T, et al. Advanced prostate cancer presenting as hemolytic uremic syndrome. Case Rep Urol 2013;2013:459-61.

8. Lechner K, Obermeier HL. Cancer-related microangiopathic hemolytic anemia: clinical and laboratory features in 168 reported cases. Medicine 2012;91:195-205.

9. Chang JC, Naqvi T. Thrombotic thrombocy- topenic purpura associated with bone marrow metastasis and secondary myelofibrosis in cancer. Oncologist 2003;8:375-80.

10. Werner TL, Agarwal N, Carney HM, Rodgers GM. Management of cancer-associated thrombotic microangiopathy: what is the right approach? Am $\mathrm{J}$ Hematol 2007;82:295-8.

11. Elliott MA, Letendre L, Gastineau DA, et al. Cancer-associated microangiopathic hemolytic anemia with thrombocytopenia: an important diagnostic consideration. Eur J Haematol 2010;85:43-50.

12. Alonso JV, Fonseca J, Lopera EL, et al. A report of disseminated adenocarcinoma presenting as thrombotic thrombocytopenic purpura. Hematol Rep 2011;3:e14.

13 Shin SY, Park H, Chae SW, Woo HY. Microangiopathic hemolytic anemia as the first manifestation of metastatic signet ring cell carcinoma of unknown origin: a case report and review of literature. Korean J Lab Med 2011;31:157-61.

14 Francis KK, Kalyanam N, Terrell DR, et al. Disseminated malignancy misdiagnosed as thrombotic thrombocytopenic purpura: A report of 10 patients and a systematic review of published cases. Oncologist 2007;12:11-9.

15 Hodgson K, Ferrer G, Pereira A, et al. Autoimmune cytopenia in chronic lymphocytic leukaemia: diagnosis and treatment. Br J Haematol 2011;154:14-22.

16 Moreno C, Hodgson K, Ferrer G, et al. Autoimmune cytopenia in chronic lymphocytic leukemia: prevalence, clinical associations, and prognostic significance. Blood 2010;116:4771-6.

17 Spira MA, Lynch EC. Autoimmune hemolytic anemia and carcinoma: an unusual association. Am J Med 1979;67: 753-8.

18 Puthenparambil J, Lechner K, Kornek G. Autoimmune hemolytic anemia as a paraneoplastic phenomenon in solid tumors: a critical analysis of 52 cases reported in the literature. Wien Klin Wochenschr 2010;122:229-36.

19. Kamesaki T, Toyotsuji T, Kajii E. Characterization of direct antiglobulin test-negative autoimmune hemolytic anemia: a study of 154 cases. Am J Hematol 2013;88:93-6. 